similar pattern of fracture (Roy-Camille et al. 1985), while stress fractures of the sacrum have been reported after radiotherapy for pelvic tumours (Cooper, Beabout and Swee 1985).

Stress fracture of the sacrum is not a common cause of low back pain, but should be considered in osteoporotic patients with appropriate symptoms and signs, since an early diagnosis may obviate unnecessary diagnostic and therapeutic measures.

\section{REFERENCES}

Cooper KL, Beabout JW, Swee RG. Insufficiency fractures of the sacrum. Radiology 1985:156:15-20.

Freeman MAR, Todd RC, Pirie CJ. The role of fatigue in the pathogenesis of senile femoral neck fractures. J Bone Joint Surg [Br] 1974:56-B:698-702.

Ries T. Detection of osteoporotic sacral fractures with radionuclides. Radiology 1983:146:783-5.

Roy-Camille R, Saillant G, Gagna G, Mazel C. Transverse fracture of the upper sacrum: suicidal jumper's fracture. Spine $1985: 10: 838-45$

\title{
THE ELBOW FAT PAD SIGN: BRIEF REPORT
}

\author{
D. N. QUINTON, D. FINLAY, R. BUTTERWORTH
}

The fat pad signs of effusion into the elbow joint consist of two translucent areas seen on a lateral radiograph (Fig. 1): the posterior area (not seen in a normal joint) lies behind the intercondylar fossa of the humerus; the anterior becomes "sail-shaped" when displaced. The relationship of positive fat pad signs to the presence of a fracture has been the subject of controversy (Norrell 1954: Kohn 1959; Murphy and Siegel 1977: Hall-Craggs, Shorvon and Chapman 1985). We report a study of patients with elbow trauma who have radiological effusions but no fracture on the initial radiograph.

Material and methods. All patients presenting over a sixmonth period with trauma to the elbow sustained less than 72 hours previously were assessed. Those with pain, tenderness or limited movement had radiographs taken of the elbow: an anteroposterior view with the forearm in mid-pronation, and a lateral view in $90^{\circ}$ of flexion.

If no sign of effusion or fracture was seen on the radiograph and there was minimal discomfort, the patient was discharged. Patients with fractures were referred to the fracture clinic for treatment. Those without fractures but with positive fat pad signs of effusion had oblique views taken of the radial head in pronation and supination. They were treated in a broad arm sling until the pain had subsided, and were followed up until they had regained full painless movement. In these cases repeat radiographs were taken two or three weeks later to ensure the absence of fracture. In those patients with persistent fat pad signs or persistent discomfort further films were subsequently obtained. All radiographs were later reviewed by a consultant radiologist.

Results. During the period of the study, 820 patients had radiographs of the elbow taken for trauma. Of these, 244

D. N. Quinton, FRCS, Senior Registrar in Accident and Emergency D. Finlay, DRCR, FRCP, Consultant Radiologist

R. Butterworth, MBChB, Senior House Officer in Accident and Emergency

The Leicester Royal Infirmary, Leicester LEI 5WW, England.

Correspondence to Mr D. N. Quinton.

(C) 1987 British Editorial Society of Bone and Joint Surgery $0301-620 \mathrm{X} / 87 / 5 R 34 \$ 2.00$

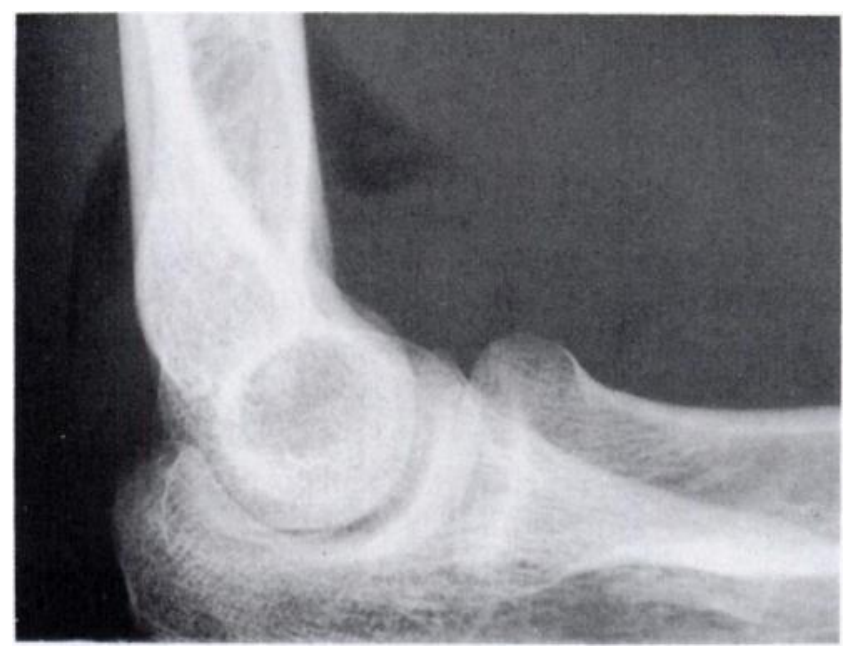

Fig. 1

were initially diagnosed as having fractures, and 56 had positive fat pad signs of effusion but no obvious fracture. All of these patients had a positive anterior fat pad sign with a mean size of $8 \mathrm{~mm}$ (range 4 to $13 \mathrm{~mm}$ ); 34 patients also had a positive posterior sign with a mean size of $2.1 \mathrm{~mm}$ (range 1 to $7 \mathrm{~mm}$ ). There was no relationship between the size of the anterior fat pad and the presence, absence or size of the posterior fat pad.

At review of those with positive fat pad signs four were shown to have loose bodies in the joint, and one proved to have had a pulled elbow. Of the 51 patients in whom no obvious reason for effusion could be diagnosed, only six were later shown to have minor fractures (four radial head, one radial neck and one supracondylar); all six recovered rapidly. At the second review (at three weeks) most of the 45 remaining patients had a normal range of movement, with a mean loss of $5^{\circ}$ of flexion and/or extension. Eleven patients had persistence of positive fat pad signs, mainly anterior, and these were more likely to have discomfort and limited movement in the elbow at that stage. All 45 patients eventually recovered a full painless range of movement at between 14 and 38 days after injury. 
Discussion. We agree with most other observers that positive fat pad signs indicate an effusion. In our series the presence of an effusion without a fracture on the initial radiograph equated with a rapid return to normal function. In only six cases was the effusion the only sign of a fracture which became apparent later, compared with the 45 patients with an effusion who had no demonstrable fracture even later. We therefore suggest that in patients who have injured the elbow a positive fat pad sign in isolation is not pathognomonic of a fracture and that unless there are any clinical indications the finding can be ignored.

\section{REFERENCES}

Hall-Craggs MA, Shorvon PJ, Chapman M. Assessment of the radial head - capitellum view and the dorsal fat-pad sign in acute elbow trauma. AJR 1985:145:607-9.

Kohn AM. Soft tissue alterations in elbow trauma. $A J R$ 1959:82:867-74.

Murphy WA, Siegel MJ. Elbow fat pads with new signs and extended differential diagnosis. Radiology 1977:124:659 65.

Norrell H-G. Roentgenologic visualizations of extracapsular fat: its importance in diagnosis of traumatic injuries to the elbow. Acta Radiol 1954:42:205-10.

\title{
TREATMENT OF EARLY-ACUTE OSTEOMYELITIS IN CHILDHOOD: BRIEF REPORT
}

\author{
W. G. COLE
}

Acute haematogenous osteomyelitis may present in childhood in one of two ways. In the milder early form there is a febrile illness of less than $\mathbf{4 8}$ hours' duration, localised pain, tenderness and swelling, but no abscess; in the more severe late form, there is a more prolonged illness and clinical evidence of an abscess (Cole, Dalziel and Leitl 1982). Our previous study showed that children with early-acute osteomyelitis were cured by a six-week course of antibiotics without operation and with less than one week in hospital. Antibiotics were given intravenously for a few days, then oral treatment was started in hospital and continued at home. We have now shown that a three-week course of antibiotics and immobilisation is equally successful.

The protocol. Apart from halving the duration of antibiotic treatment, the protocol for this study was that reported previously (Cole et al. 1982). All children are admitted to hospital and standard radiographs, haematological tests and blood cultures are obtained. Immediate treatment is with flucloxacillin $200 \mathrm{mg} / \mathrm{kg} / \mathrm{day}$, and intravenous benzylpenicillin six-hourly in a dose varying from 250000 to one million units. If the child is known to be allergic to penicillin then cephalothin is given. When the antibiotic sensitivities of the pathogen become known, a single appropriate antibiotic is selected. This is usually flucloxacillin for Staphylococcus aureus and benzylpenicillin for Group A $\beta$-haemolytic streptococci. If bacteria are not isolated, both of the antibiotics used initially are continued.

Intravenous antibiotics are given until the child is clinically well, with less fever and some decrease in the local signs. At this stage, flucloxacillin, phenoxymethylpenicillin or cefaclor are given by mouth. Provided that they are well and tolerate the oral antibiotics, the

W. G. Cole, MSc, PhD, FRACS, Deputy-Chief Orthopaedic Surgeon Department of Orthopaedic Surgery, The Royal Children's Hospital, Flemington Road, Parkville, Victoria 3052, Australia.

(C) 1987 British Editorial Society of Bone and Joint Surgery $0301-620 X / 87 / 5 R 32 \$ 2.00$ children return home two days later, returning for review after three weeks of treatment. The area involved is immobilised in a sling or plaster slab, which is converted to a full plaster before the child is allowed home. At the three-week review, the plaster is removed, and clinical, haematological and radiological examinations are repeated. Review continues until radiographic healing has occurred.

Patients. Fifty consecutive children with their first attack of early-acute haematogenous osteomyelitis were included. Their age and sex distributions were the same as in our previous study (Cole et al. 1982), as was the skeletal distribution. Cases of spinal osteomyelitis and discitis were excluded. The diagnosis was confirmed radiographically in all cases by the appearance of subperiosteal new bone or other changes at some stage during the course of the illness.

Bacteriology. Positive blood cultures were obtained from 27 children. Staphylococcus aureus was grown in 25, and was sensitive to flucloxacillin but resistant to benzylpenicillin in all cases. In two cases Group A $\beta$-haemolytic streptococci, sensitive to benzylpenicillin, were found. Results. All children had a rapid response to treatment within the first 24 to 48 hours of intravenous antibiotic treatment. Ten children, mostly of pre-school age, refused to take flucloxacillin syrup; they were given cefaclor as an alternative. All children returned home four to five days after admission.

After three weeks' treatment, all children had clinical recovery with an ESR which was commonly normal or had improved substantially. Radiographs showed only a fine line of subperiosteal new bone and osteoporosis, with no evidence of continuing infection. When reviewed three to four years later, well beyond the seven-month period during which most recurrences are reported (Blockey and Watson 1970), all 50 children were found to be clinically and radiologically normal.

Conclusions. Children with early-acute osteomyelitis can be cured by a few days treatment with intravenous 